\title{
Évaluation des effets dans le domaine de l'éducation à la sexualité au primaire : Exemple tiré d'une évaluation d'un programme de prévention de la sexualisation précoce
}

\author{
Éliane Dussault et Francine Duquet \\ Département de sexologie, Université du Québec à Montréal
}

\begin{abstract}
Résumé : Au Québec, des contenus en éducation à la sexualité ont été rendus obligatoires pour les élèves du préscolaire au $5^{e}$ secondaire en 2018. Des programmes en éducation à la sexualité dont l'efficacité a été évaluée sont donc de mise dans une optique de pratiques avisées de promotion de la santé. Peu de ces programmes ont fait l'objet d'évaluations des effets publiées, et ce, particulièrement dans un cadre scolaire primaire. Cet article explore les réflexions liées à l'évaluation des effets de programmes en éducation à la sexualité au primaire, en se basant sur le cas d'une évaluation d'un programme de prévention de la sexualisation précoce.
\end{abstract}

Mots-clés : éducation à la sexualité au primaire, évaluation des effets, prévention de la sexualisation précoce

Abstract: In Quebec, sexuality education contents were made mandatory for students from preschool to Secondary V in 2018. Sexuality education programs whose efficiency has been evaluated should be implemented in the context of informed health promotion practices. However, few of these programs have actually been evaluated, with published results, particularly within an elementary school framework. This article reflects on the evaluation of elementary sexuality education programs, based on a case relating to the evaluation of an early sexualization prevention program.

Keywords: elementary sexuality education, effect assessment, early sexualization prevention

\section{CONTEXTE ACTUEL DE L'ÉDUCATION À LA SEXUALITÉ AU PRIMAIRE ET DES ÉVALUATIONS DES EFFETS DES PROGRAMMES OFFERTS}

Depuis septembre 2018, plusieurs contenus d'éducation à la sexualité définis par le ministère de l'Éducation et de l'Enseignement supérieur (MEES, 2018b) sont obligatoires pour tous les élèves québécois du préscolaire au $5^{\mathrm{e}}$ secondaire. Ces

Correspondance à l'auteur : Éliane Dussault, dussault.eliane@courrier.uqam.ca 
contenus, présentés sous forme d’objectifs sous-divisés selon plusieurs thématiques (ex. : croissance sexuelle et image corporelle, vie affective et amoureuse), ne sont pas enseignés dans le cadre d'une matière qui leur est consacrée, mais plutôt au travers de l’ensemble des matières déjà établies dans le cursus scolaire (MEES, 2018b). Ainsi, des ressources, dont des programmes d'éducation à la sexualité, sont proposées pour atteindre les objectifs d'apprentissage. Or, des outils dont l'efficacité a été évaluée sont bénéfiques aux démarches de promotion de la santé (Palluy et coll., 2010), tout comme l'est l'éducation à la sexualité en milieu scolaire. De plus, l'efficacité des pratiques de promotion de la santé repose sur des recherches évaluatives démontrant l'efficience de programmes (Palluy et coll., 2010). Par ailleurs, les Lignes directrices canadiennes pour l'éducation en matière de santé sexuelle (Agence de la santé publique du Canada, 2008) tentent de guider et d'unifier les professionnels dans les milieux où se transmet l'éducation à la sexualité, et ce, en suggérant aux planificateurs un processus de base en trois étapes : 1) l'évaluation des besoins en matière de santé sexuelle, 2) lélaboration des interventions en matière de santé sexuelle et 3) la mesure de l'efficacité du programme ainsi que la détermination des éléments devant être modifiés.

Dans un autre ordre d'idées, l'éducation à la sexualité est, de façon assez consensuelle, perçue comme bénéfique pour les adolescents, mais celle prodiguée aux enfants pourrait, selon certains, priver ceux-ci de leur innocence ou ne pas leur être bénéfique (MEES, 2018a). Pourtant, les enfants sont tous sexués et ont des besoins auxquels il faut répondre en matière déducation à la sexualité (ex. : ont des questionnements, découvrent leur identité sexuelle, ont des sources d'information contradictoires quant à la sexualité) (MEES, 2018a). De plus, les orientations internationales en éducation sexuelle de l'Organisation des Nations Unies pour l'éducation, la science et la culture (UNESCO, 2010) déclarent que les enfants doivent développer des compétences dès un jeune âge leur permettant de comprendre non seulement leur corps, mais aussi leurs relations aux autres et leurs sentiments, en fonction de leur niveau de développement. Finalement, lère actuelle de l'éducation à la sexualité se veut holistique, inclusive et positive, ce qui implique de respecter quelques principes fondamentaux, tels que l'encouragement à la participation des jeunes, le développement de leurs compétences de réflexion critique ou encore, la prise en compte de facteurs liés aux vulnérabilités et à l'exclusion (Fonds des nations unies pour la population [UNFPA], 2015). Ces éléments doivent également être tenus en compte lors de l'évaluation des effets des programmes dans le domaine de l'éducation à la sexualité (UNFPA, 2015). Tout cela fait en sorte que cette problématique est démontrée comme ayant une envergure sociale, et en justifie la prise en charge par des évaluations de programmes (Rossi, Lipsey et Howard, 2004).

Dans cette optique où l'évaluation des effets des programmes en éducation à la sexualité doit être documentée, dans le contexte de l'implantation des contenus obligatoires en éducation à la sexualité au Québec, et à l'ère de l'éducation à la sexualité positive et complète, peu de réflexions ont, à ce jour, été engendrées en matière de pratiques évaluatives gagnantes dans le domaine de l'éducation à la sexualité au sein des milieux scolaires primaires. Dans la mesure où une place 
à l'importance croissante et plus formelle est accordée à l'éducation à la sexualité en milieu scolaire primaire, il appert d'enrichir les connaissances au niveau du processus dévaluation des programmes dispensés dans ce cadre. Les objectifs de l'article sont : 1) de documenter les écrits dans le domaine de léducation à la sexualité et de l'évaluation des effets de programmes au primaire ainsi que 2) d'illustrer et d'enrichir ces réflexions à l'aide d'un cas d'évaluation des effets d'un programme d'éducation à la sexualité au primaire.

\section{Synthèse des évaluations des effets de programmes d'éducation à la sexualité au primaire}

Quoique les évaluations des effets de programmes deéducation à la sexualité au primaire soient peu documentées, voire peu nombreuses jusquà présent, certaines d'entre elles permettent de guider des choix théoriques et méthodologiques à privilégier pour les futures évaluations. Ces programmes, destinés à des enfants du primaire et ayant fait l'objet d'une évaluation des effets, semblent la plupart du temps inclure un groupe expérimental ainsi qu'un groupe témoin à la recherche (Bourassa et Rousseau, 2004; Donnelly, Horn, Young et Ivanescu, 2016; Hébert, Lavoie, Piché et Poitras, 1999; Miller et coll., 2017; Verlaan, Charbonneau et Turmel, 2005). Les mesures dévaluation varient : certaines sont des questionnaires de type « vrai ou faux» ou " en accord/en désaccord » sur le contenu du programme visant à mesurer l'acquisition de connaissances (Bourassa et Rousseau, 2004; Donnelly et coll., 2016; Hébert et coll., 1999; Verlaan et coll., 2005), tandis que d'autres utilisent des questionnaires portant sur des variables (ex. : comportementales) sur lesquelles on souhaite voir un changement entre les mesures pré-/post-test (Miller et coll., 2017; Tremblay et Bégin, 2008; Verlaan et coll., 2005).

Les programmes présentent souvent des différences significatives entre les groupes expérimentaux et témoins de la recherche (Bourassa et Rousseau, 2004; Donnelly et coll., 2016; Hébert et coll., 1999; Miller et coll., 2017; Verlaan et coll., 2005). Des mesures pré- et post-test permettent de déterminer qu'un changement au niveau des scores soit bel et bien attribuable au fait d'avoir assisté au programme et non à un autre facteur (Bourassa et Rousseau, 2004; Donnelly et coll., 2016; Hébert et coll., 1999; Miller et coll., 2017; Verlaan et coll., 2005). Le post-test semble se mesurer immédiatement après la fin du programme (Bourassa et Rousseau, 2004; Miller et coll., 2017; Tremblay et Bégin, 2008). Certaines mesures d'implantation du programme sont parfois utilisées lors d'évaluations des effets, notamment en ce qui concerne la formation reçue par les enseignants au préalable de l'animation du programme (Donnelly et coll., 2016; Miller et coll., 2017) et l'appréciation du programme par la clientèle cible (Bourassa et Rousseau, 2004; Mayne, 2015; Verlaan et coll., 2005; Stake, 1967).

\section{Exemple de l'évaluation du programme « On est encore des enfants! »}

Le programme « On est encore des enfants! » (Duquet, 2017) a été conçu afin de répondre à des préoccupations de professionnels intervenant auprès des enfants 
âgés de 10 à 12 ans ( $5^{\mathrm{e}}$ et $6^{\mathrm{e}}$ années du primaire), en réponse aux conséquences observées de la sexualisation précoce sur leur développement. La sexualisation précoce est définie comme une exposition des enfants à des formes stéréotypées de sexualité adulte, résultant de publicités et de marketing ne respectant pas leur niveau de développement psychosexuel qui, au lieu de sopérer de façon lente et progressive, s'en retrouve accéléré (Rush et La Nauze, 2006).

La conception du programme a été effectuée dans le cadre d'une démarche de recherche-intervention. La version finale du programme a été éditée à la suite d'une démarche de validation et d'évaluation de son implantation (non publiée). En ce sens, une première version a été créée à la suite d'une recherche exploratoire réalisée auprès d'enfants âgés de 10 à 12 ans afin de dresser un portrait de ce à quoi ils étaient confrontés en lien avec le phénomène de sexualisation précoce (ex. : pression à avoir un amoureux ou une amoureuse, exposition à la pornographie, etc.) (Duquet, 2008a) ainsi qu'à la suite des besoins d'intervention exprimés par du personnel scolaire au primaire (Duquet, 2008b). De ces études exploratoires se sont dégagées des pistes d'intervention d'où ont émergé les thématiques du programme. Ce dernier a fait l'objet d'une validation, dont : 1) une lecture critique de la part de 10 intervenants œuvrant auprès des enfants et 2) une bonification à la suite de leurs commentaires. Puis, une expérimentation a été réalisée, ce qui a permis des bonifications supplémentaires avant son édition finale. Le programme final a pour intention pédagogique générale de « Protéger le droit à l'enfance, tout en offrant aux enfants des balises vis-à-vis de situations inappropriées pour leur âge en matière de sexualité » (Duquet, 2017, p. 10). Il est à noter qu'il comprend des activités obligatoires à l'atteinte des objectifs ainsi que des activités complémentaires. Dans le cadre de la recherche, seules les activités obligatoires ont été implantées et évaluées. Finalement, quoiqu'aucune théorie n'ait servi d'ancrage à la conception du programme et que cela doive être considéré comme l'une de ses limites, l'ensemble du processus de conception, selon Duquet (2008a, 2017), fait en sorte que les thématiques du programme sont en lien avec la prévention de la sexualisation précoce.

\section{Modèle d'évaluation}

Le modèle utilisé pour lévaluation du programme était le Countenance Model de Stake (1967). Ce dernier comporte une matrice descriptive des éléments à considérer dans l'évaluation de programme (ex. : activités, personnes rejointes, préalables à la réalisation de l'évaluation, etc.). Une deuxième matrice permet de poser le jugement de lévaluation en fonction de critères et de standards découlant de la description du programme effectuée préalablement (Stake, 1967). Ce modèle tient également compte de l'implantation du programme ainsi que des effets inattendus qui pourraient être produits par le fait d'avoir suivi le programme (Stake, 1967). Dans le cadre de l'article, nous nous limiterons à expliquer une partie des résultats seulement, en regard des réflexions sur l'évaluation de programme qu'ils ont suscitées. Cependant, en respect du modèle d'évaluation sélectionné, une évaluation plus complète de ce programme a été réalisée et inclut des mesures d'implantation 
du programme et ses potentiels effets inattendus (Dussault, 2018). Par ailleurs, quoiquà notre connaissance peu de programmes déducation à la sexualité des enfants aient utilisé ce modèle dévaluation, plusieurs études évaluatives portant sur des programmes d'éducation aux enfants et/ou conduites en milieu scolaire ont utilisé ce modèle (Chafel, 1981; Fatima, Malik et Abid, 2016; Thanabalan, Siraj et Alias, 2015). Celui-ci semble donc intéressant à considérer dans le cadre des évaluations des effets de programmes d'éducation à la sexualité au primaire.

\section{Objet de l'évaluation}

Le présent article porte sur une partie de l'évaluation du programme « On est encore des enfants! » (Duquet, 2017). Celui-ci a été évalué en matière de ses effets à court terme sur les connaissances des enfants à l'égard des thèmes du programme.

\section{MÉTHODOLOGIE}

Le devis d'évaluation utilisé pour la recherche est un devis pré-post avec comparaison indépendante (Haccoun et McDuff, 2009), incluant un groupe expérimental et un groupe témoin à la recherche. Puisque les participants nont pas été sélectionnés aléatoirement (l'échantillonnage utilisé était de convenance), le devis est de type quasi expérimental (Haccoun et McDuff, 2009). Au sein du Countenance Model de Stake (1967), l'évaluation d'un programme doit, entre autres, porter un jugement sur les effets de l'enseignement sur les apprentissages en contrôlant les potentiels effets inattendus de ce programme, alors que ce choix de devis d'évaluation permet de porter un tel type de jugement. En effet, le devis de recherche sélectionné permet d'estimer la cause probable du résultat de l'évaluation, en plus de démontrer que s'il existe une différence pré-post, celle-ci ne soit constatée que pour le groupe qui a participé à l'intervention (Haccoun et McDuff, 2009). Le choix du devis méthodologique semble donc bien s'arrimer au choix du modèle théorique de l'évaluation réalisée.

\section{Participants}

Les participants à létude sont 77 enfants âgés de 10 à 12 ans $\left(5^{\mathrm{e}}\right.$ et $6^{\mathrm{e}}$ années primaire) parlant couramment le français. Un groupe de $5^{\mathrm{e}}$ année et un groupe de $6^{\mathrm{e}}$ année ont été recrutés pour composer les groupes-classes expérimentaux. Un groupe de $5^{\mathrm{e}}$ année et un groupe de $6^{\mathrm{e}}$ année ont également été recrutés en tant que groupes témoins. Les quatre groupes-classes ont été recrutés parmi quatre écoles d'une commission scolaire québécoise, dont les rangs déciles des indices de milieu socio-économique pour l'année 2016-2017 étaient les suivants : $5^{\mathrm{e}}$ année expérimental $=3 ; 5^{\mathrm{e}}$ année témoin $=1 ; 6^{\mathrm{e}}$ année expérimental $=9 ; 6^{\mathrm{e}}$ année témoin $=8$ (1 étant le plus favorisé et 10 étant le plus défavorisé) (MEES, 2017).

Les enseignantes des deux groupes-classes expérimentaux se sont portées volontaires à l'implantation du programme dans leur groupe classe respectif. Ces deux enseignantes ont été recrutées par le biais des gestionnaires d'une commission scolaire québécoise. Ces dernières avaient déjà suivi une formation de deux 
jours portant sur la problématique de sexualisation précoce de même que sur l'appropriation du programme préalablement à l'expérimentation de la recherche. La méthode d'échantillonnage est donc dite de convenance. En effet, les participants sélectionnés sont ceux ayant fréquenté une école primaire faisant partie de la commission scolaire et ayant accepté de participer au projet de recherche. Le tableau 1 indique le détail des participants recrutés dans le cadre de l'étude.

Le processus de recrutement s'est effectué en plusieurs étapes. D’abord, le projet de recherche a été présenté, puis approuvé par le comité d'éthique de la recherche pour les projets étudiants (CERPÉ) de l'Université du Québec à Montréal (UQAM). Puis, l'accord de la direction de la commission scolaire à ce que des enseignantes et des enfants participent au projet de recherche a été obtenu. Ensuite, le projet a été soumis aux directions d'école qui ont aussi donné leur accord pour que les enseignantes soient formellement recrutées, de même que les élèves faisant partie de leurs groupes-classes. Puis, par l'intermédiaire des enseignantes sélectionnées sur une base volontaire (groupe expérimental et groupe témoin), un formulaire de consentement a été envoyé aux parents des élèves, afin qu'ils fournissent leur accord à ce que leur enfant participe à l'étude. Pour terminer, les enfants souhaitant participer à l'étude ont fourni leur accord à l'aide d'un formulaire de consentement.

\section{Procédure de collecte}

Les données ont été recueillies auprès de deux groupes indépendants, dont l'un a bénéficié de l'intervention, et l'autre pas (Haccoun et McDuff, 2009). Plus précisément, deux groupes-classes ont formé le groupe expérimental et deux groupesclasses ont formé le groupe témoin. Le groupe expérimental a réalisé les activités

Tableau 1 : Détail des participants recrutés

\begin{tabular}{llr}
\hline Groupe & Participants & $\mathbf{n}$ \\
\hline Expérimental $5^{\mathrm{e}}$ année & Enfants & 23 \\
& Filles & 12 \\
& Garçons & 11 \\
Expérimental 6e année & Enseignante animatrice & 1 \\
& Enfants & 16 \\
& Filles & 9 \\
Témoin $5^{e}$ année & Garçons & 7 \\
& Enseignante animatrice & 1 \\
Témoin 6e année & Enfants & 15 \\
& Filles & 9 \\
& Garçons & 6 \\
& Enfants & 23 \\
& Filles & 12 \\
\hline
\end{tabular}


comprises parmi les trois thématiques destinées à son degré scolaire $-5^{\mathrm{e}}$ ou $6^{\mathrm{e}}$ année. Le groupe témoin a suivi le cursus habituel offert en matière d'éducation à la sexualité au sein de cette commission scolaire. Les tableaux $2 \mathrm{a}$ et $2 \mathrm{~b}$ présentent le détail de la procédure de collecte de données. Précisons quen ce qui concerne les durées d'implantation des activités, celles-ci incluent la passation du questionnaire post-test (d'une durée de 5 à 10 minutes) à la suite des rencontres concernant les groupes-classes expérimentaux.

L'expérimentation de la recherche s'est effectuée au printemps 2017. Les groupes de recherche ont été sélectionnés dans des écoles différentes afin de minimiser les contacts entre les élèves de différents groupes. D'un mois à un mois et demi sest écoulé entre la passation du prétest et du post-test final chez tous les groupes. Un questionnaire prétest a été rempli par les enfants environ 2 semaines avant l'implantation du programme. Le questionnaire post-test a été rempli à la suite de chacune des thématiques dispensées chez les participants du groupe expérimental, tandis qu'il a été rempli un mois après le premier temps de mesure chez les participants du groupe témoin. Pour les groupes expérimentaux, le questionnaire post-test a été subdivisé selon les trois thématiques offertes pour chaque degré (pour un total de trois questionnaires post-tests distribués lors des trois demi-journées d'expérimentation du programme). Chacune des thématiques destinées à un degré $-5^{\mathrm{e}}$ ou $6^{\mathrm{e}}$ année - était donc implantée lors d'une même demi-journée chez ces groupes, alors que le questionnaire post-test était rempli immédiatement après la tenue de l'expérimentation d’une thématique.

\section{Mesures}

Les données ont été recueillies à l'aide d'une fiche signalétique mesurant certaines variables sociodémographiques de même quà l'aide de questionnaires prétest et

Tableau 2a : Éducation à la sexualité reçue chez les élèves de $5^{e}$ année

\begin{tabular}{lll}
\hline & $\mathbf{5}^{\mathbf{e}}$ année expérimental & $\mathbf{5}^{\mathbf{e}}$ année témoin \\
\cline { 2 - 3 } & Dates d'implantation & \\
\hline Ateliers donnés par l'infirmière & $\mathrm{n} / \mathrm{a}$ & $\mathrm{n} / \mathrm{a}$ \\
Questionnaire prétest & 27 mars & $4 \mathrm{avril}$ \\
& $1 \mathrm{~h}$ & $1 \mathrm{~h}$ \\
Rencontre 5.1 & 10 avril & $\mathrm{n} / \mathrm{a}$ \\
Rencontre 5.2 & $3 \mathrm{~h}$ & $\mathrm{n} / \mathrm{a}$ \\
Rencontre 5.3 & 25 avril & \\
Questionnaire post-test & $2,5 \mathrm{~h}$ & $\mathrm{n} / \mathrm{a}$ \\
Total : & $12 \mathrm{mai}$ & $4 \mathrm{mai}$ \\
& $2,5 \mathrm{~h}$ & $1 \mathrm{~h}$ \\
\hline
\end{tabular}


Tableau 2b : Éducation à la sexualité reçue chez les élèves de $6^{e}$ année

\begin{tabular}{|c|c|c|}
\hline & $6^{e}$ année expérimental & $6^{e}$ année témoin \\
\hline & \multicolumn{2}{|l|}{ Dates d'implantation } \\
\hline Ateliers donnés par & 17 mars & 16,20 et 28 mars; 7 avril \\
\hline l'infirmière & $2,5 \mathrm{~h}$ & $6 \mathrm{~h}$ \\
\hline Questionnaire prétest & 22 mars & 31 mars \\
\hline & $1 \mathrm{~h}$ & $1 \mathrm{~h}$ \\
\hline Rencontre 6.1 & $\begin{array}{l}3 \text { avril } \\
4 \mathrm{~h}\end{array}$ & $\mathrm{n} / \mathrm{a}$ \\
\hline Rencontre 6.2 & $\begin{array}{l}25 \text { avril } \\
2,5 \mathrm{~h}\end{array}$ & $\mathrm{n} / \mathrm{a}$ \\
\hline Rencontre 6.3 & $\begin{array}{l}28 \text { avril } \\
2,5 \mathrm{~h}\end{array}$ & $\mathrm{n} / \mathrm{a}$ \\
\hline Questionnaire post-test & $\mathrm{n} / \mathrm{a}$ & $\begin{array}{l}28 \text { avril } \\
1 \mathrm{~h}\end{array}$ \\
\hline Total : & Environ $13 \mathrm{~h}$ & Environ $7 \mathrm{~h}$ \\
\hline
\end{tabular}

post-test, formulés pour chacun des degrés ( $5^{\mathrm{e}}$ et $6^{\mathrm{e}}$ années). Les questionnaires ont été conçus à partir du contenu (ex. : messages-clés, diaporamas, etc.) du programme. Les participants devaient répondre à des affirmations de type "vrai ", " faux » ou " je ne sais pas ", afin de vérifier la justesse de leurs connaissances à l'égard de chacun des contenus au prétest et au post-test (ex. : " Un adolescent a plus de privilèges et de permissions qu'un enfant » ou «Les enfants peuvent avoir le même genre d'histoire d'amour que les adultes »). Les scores aux tests de connaissances cumulent les énoncés couverts par les questionnaires reprenant le contenu des rencontres couvertes, et représentent un point par bonne réponse à un item. Pour les enfants de $5^{\mathrm{e}}$ année, le score maximal aux tests est de 41 points, tandis qu'il est de 47 points chez ceux de 6 année. Les instruments de mesure ont été conçus par l'auteure principale et validés par un comité d’experts. Une mesure de la cohérence interne pour chacun des questionnaires révèle des coefficients satisfaisants en $5^{\mathrm{e}}$ année (pré : $\alpha=0,80$ et post : $\alpha=0,84$ ) autant quen $6^{\mathrm{e}}$ année (pré : $\alpha=0,92$ et post $: \alpha=0,92$ ).

\section{Analyses}

Des analyses de chi-deux dressent un portrait de léchantillon. Des analyses de covariance (ANCOVAs) à mesures répétées ont été effectuées afin de mesurer l'effet du temps et du programme chez les participants aux deux temps de mesure de même que de situer à quel facteur se situerait une éventuelle différence significative entre les groupes. La variable indépendante est constituée des scores aux deux temps de mesure (pré/post), tandis que la variable dépendante est composée du groupe - expérimental ou témoin - de la recherche. De plus, un contrôle de certaines variables sociodémographiques permet de cibler si lesdites différences 
peuvent s'expliquer par ces dernières. En matière des variables de contrôle, seules celles ayant un effet significatif sont conservées dans le modèle final. Les variables qui ont été testées sont le genre (garçon ou fille), les résultats scolaires $(1=$ très bonnes notes $[80 \%$ et plus] à $3=$ pas de bonnes notes [ $60 \%$ et moins]), l'importance accordée à la réussite scolaire $(0=$ pas du tout important à $2=$ très important) ainsi que l'indice de milieu socio-économique (de 1 à 10, 1 étant le plus favorisé et 10 étant le plus défavorisé) (MEES, 2017).

\section{RÉSULTATS}

\section{Portrait de l'échantillon}

Le tableau 3 illustre que, pour l'ensemble des enfants participant à l'étude, environ la moitié $(48,7 \%)$ est en $5^{\mathrm{e}}$ année, tandis que l'autre moitié $(51,3 \%)$ d'entre eux est en $6^{\mathrm{e}}$ année. Lâge moyen des participants est de 10,95 ans $(\mathrm{ET}=0,72)$. Les groupes expérimentaux et témoins sont respectivement composés de $53,8 \%$ et $54,1 \%$ de filles. Aucune différence significative n'est présente entre le groupe expérimental et témoin de la recherche au niveau des variables analysées $(p>0,05)$.

Tableau 3 : Portrait de l'échantillon

\begin{tabular}{|c|c|c|c|c|}
\hline & \multicolumn{4}{|c|}{ Pourcentage (\%) } \\
\hline & Total & Expérimental & Témoin & Sig. \\
\hline \multicolumn{5}{|l|}{ Degré scolaire } \\
\hline 5e année & 48,7 & 56,4 & 40,5 & \multirow[t]{2}{*}{0,167} \\
\hline 6e année & 51,3 & 43,6 & 59,5 & \\
\hline \multicolumn{5}{|l|}{ Genre de l'enfant } \\
\hline Féminin & 53,9 & 53,8 & 54,1 & \multirow[t]{2}{*}{0,985} \\
\hline Masculin & 46,1 & 46,2 & 45,9 & \\
\hline \multicolumn{5}{|l|}{ Âge } \\
\hline 10 ans & 25,0 & 28,2 & 21,6 & \multirow{4}{*}{0,643} \\
\hline 11 ans & 52,6 & 51,3 & 54,1 & \\
\hline 12 ans & 21,1 & 17,9 & 24,3 & \\
\hline 13 ans & 1,3 & 2,6 & 0,0 & \\
\hline \multicolumn{5}{|l|}{ Résultats scolaires } \\
\hline $60 \%$ et moins & 4,0 & 7,7 & 0,0 & \multirow{3}{*}{0,059} \\
\hline $70 \%$ & 41,3 & 30,8 & 52,9 & \\
\hline $80 \%$ et plus & 54,7 & 61,5 & 47,2 & \\
\hline \multicolumn{5}{|c|}{$\begin{array}{l}\text { Importance accordée à la réussite } \\
\text { scolaire }\end{array}$} \\
\hline Pas du tout importante & 1,3 & 2,6 & 0,0 & \multirow[t]{3}{*}{0,512} \\
\hline Un peu importante & 3,9 & 2,6 & 5,4 & \\
\hline Très importante & 94,7 & 94,9 & 94,6 & \\
\hline
\end{tabular}




\section{Effets du programme sur les connaissances des élèves de $5^{e}$ année}

Une ANCOVA à mesures répétées révèle une homogénéité de la variance pour les variables mesurées chez les $5^{e}$ années (test de Levene basé sur la moyenne $=0,27$; $p=0,607)$. Les variables indépendantes démontrent une inégalité de la covariance avec la variable dépendante (test de $\mathrm{Box}=10,00 ; p=0,029$ ). Une absence de différence significative est démontrée entre le groupe expérimental et le groupe témoin en regard du facteur de temps (comme démontré à la figure 1, il y a une baisse de score des enfants du groupe témoin entre les deux temps de mesure et une hausse de celui des enfants du groupe expérimental.). Cela indique que le temps écoulé entre les deux temps de mesure n’a pas d'effet sur les résultats des participants. Cependant, la différence en ce qui concerne la participation au programme savère significative (Trace de Pillai $=7,45 ; p=0,012$ ). Les enfants du groupe expérimental $(\mathrm{M}=29,46 ; \mathrm{ET}=5,17)$ ont une moyenne inférieure à celle du groupe témoin au prétest $(\mathrm{M}=32,72 ; \mathrm{ET}=4,50)$, mais présentent une moyenne plus élevée au posttest (respectivement, $\mathrm{M}=34,00 ; \mathrm{ET}=5,21$ et $\mathrm{M}=30,90 ; \mathrm{ET}=5,76$ ). Cela indique que le fait d'avoir assisté ou non au programme présente une différence. Le calcul de la taille d'effet démontre un grand effet entre les deux groupes pour le facteur " programme » (Eta-carré partiel $=0,25)$. Aucune variable de contrôle ne permet d'expliquer les différences significatives entre les groupes.

\section{Effets du programme sur les connaissances des élèves de $\sigma^{e}$ année}

Une ANCOVA à mesures répétées révèle une homogénéité de la variance pour les variables mesurées chez les élèves de $6^{e}$ année (test de Levene basé sur la

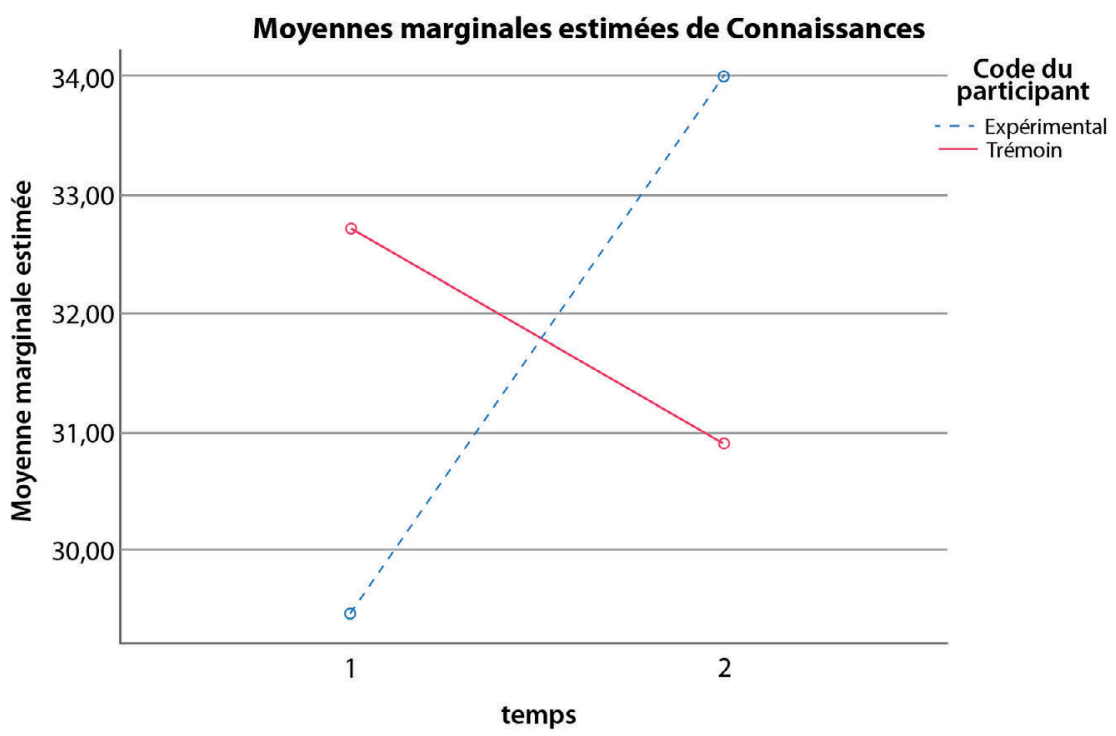

Figure 1 : Effets du programme sur les connaissances des élèves de $5^{\mathrm{e}}$ année 


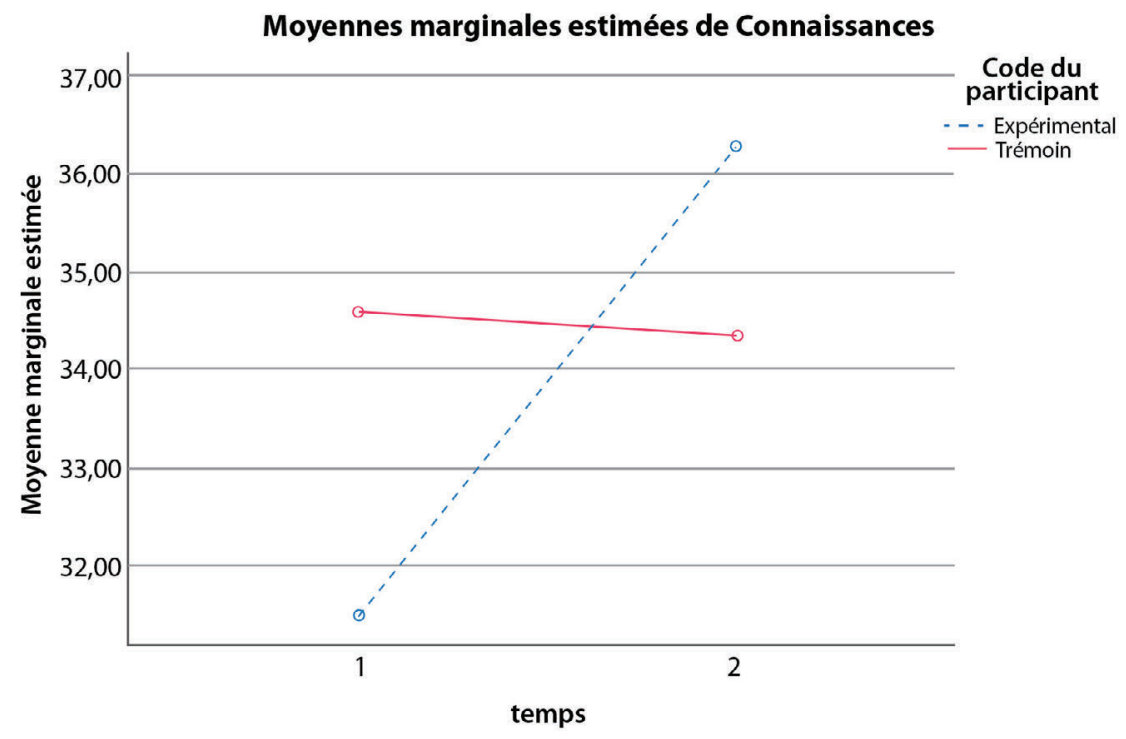

Figure 2 : Effets du programme sur les connaissances des élèves de $6^{e}$ année

moyenne $=3,51 ; p=0,071$ ). Les variables indépendantes (scores aux 2 temps de mesure) ne démontrent pas de covariance avec la variable dépendante (groupe expérimental ou témoin de la recherche) (Test de Box $=6,69 ; p=0,104$ ). Des différences significatives sont présentes entre les groupes au niveau du facteur temps (Trace de Pillai $=4,92 ; p=0,034$ ) et de l'effet du programme (Trace de Pillai $=6,08 ; p=0,020$ ). Cela signifie que l'écart de temps entre les deux mesures ainsi que le fait d'avoir ou non suivi le programme ont un effet sur le score des participants. Les enfants du groupe expérimental ont une moyenne au prétest significativement plus basse $(\mathrm{M}=31,50 ; \mathrm{ET}=11,31)$ que ceux du groupe témoin $(\mathrm{M}=34,60 ; \mathrm{ET}=7,06)$, alors qu'ils ont une moyenne significativement plus élevée au post-test (respectivement, $\mathrm{M}=36,25 ; \mathrm{ET}=10,61$ et $\mathrm{M}=34,35 ; \mathrm{ET}=7,31$ ). Leffet est fort pour le temps (Eta-carré partiel $=0,14$ ) tout comme pour le programme (Eta-carré partiel $=0,17$ ). La figure 2 démontre une augmentation du score chez les enfants du groupe expérimental à travers les deux temps de mesure, ainsi que la moyenne presque identique du groupe témoin entre les deux temps de mesure. Aucune variable de contrôle ne permet d'expliquer les différences significatives entre les groupes.

\section{DISCUSSION}

Nous proposons que les ancrages théoriques, la méthodologie et les leçons tirées de lévaluation du programme " On est encore des enfants! » (Duquet, 2017) puissent bénéficier aux prochaines évaluations des effets de programmes dans le domaine de léducation à la sexualité au primaire. 


\section{Interprétation des résultats et réflexions quant à l'évaluation de programme}

Chez les enfants de $5^{e}$ année, l'effet sur les scores des enfants est uniquement observé en matière du facteur du programme, et ce dernier est grand après calcul de la taille d'effet. Cela indique que le temps écoulé entre les deux temps de mesure n’a pas d'effet sur les scores des enfants, mais que le fait d'avoir participé au programme en présente un. Une différence significative est donc présente entre les enfants du groupe expérimental et ceux du groupe témoin, entre le prétest et le post-test, au niveau des connaissances des enfants. Chez les élèves de $6^{e}$ année, les mesures de temps comme l'effet du programme ont un grand effet sur les participants après le calcul de la taille d'effet. Ainsi, la différence significative au niveau des scores obtenus chez le groupe expérimental et le groupe témoin indique dans une bonne mesure son efficacité auprès de la clientèle cible (Bourassa et Rousseau, 2004; Donnelly et coll., 2016; Hébert et coll., 1999; Miller et coll., 2017; Verlaan et coll., 2005), quoique dans le cadre de notre étude, le questionnaire post-test ayant été rempli immédiatement après les rencontres, il faille limiter la durée de ces effets à court terme uniquement. Précisons que la taille restreinte de léchantillon pourrait également expliquer les différences de scores au prétest chez les groupes expérimental et témoin de $5^{\mathrm{e}}$ comme de $6^{\mathrm{e}}$ année, quoique dans le cas des élèves de $6^{e}$ année, le nombre d'heures supérieur en ateliers donnés par l'infirmière scolaire dans le groupe témoin puisse également expliquer cette différence. Résultant d'une recherche exploratoire réalisée auprès de personnel scolaire au primaire et d'enfants (Duquet, 2008a, 2008b), de même que d'un processus de validation incluant des mesures de ses conditions d'implantation, le programme parvient à opérer une amélioration des connaissances pouvant contribuer à la prévention de la sexualisation précoce chez les enfants ayant assisté aux rencontres du programme. La création de programmes en partenariat avec les milieux auxquels ils sont destinés à être implantés semble donc être une stratégie de choix dans l'optique de la création d'outils didactiques efficaces auprès de leur clientèle-cible. Dans cet ordre d'idées, il est nécessaire d'impliquer davantage les jeunes que ce n’est généralement le cas au sein des recherches évaluatives de programmes d'éducation à la sexualité (UNFPA, 2015). Cela leur permet notamment déliminer les obstacles à leur bienêtre sexuel et de collaborer avec l'équipe-école, le personnel du programme ainsi que d'autres acteurs locaux.

Rappelons que ce programme vient répondre aux orientations internationales en éducation sexuelle de l'UNESCO (2010), lesquelles déclarent que les enfants doivent développer des compétences dès un jeune âge, leur permettant de comprendre leur corps, leurs relations aux autres et leurs sentiments, en fonction de leur niveau de développement. Ces éléments relevant de léducation à la sexualité au primaire sont au cœur de la prévention de la sexualisation précoce, et ainsi du programme « On est encore des enfants! ». Par ailleurs, des outils dont l'efficacité a été évaluée sont d'autant plus bénéfiques à un tel type de démarche déducation à la sexualité (Palluy et coll., 2010), et ce, dans l'optique où cette problématique est démontrée comme ayant une envergure sociale justifiant sa prise en charge 
(Rossi et coll., 2004). Finalement, l'efficacité des pratiques de promotion de la santé repose sur des recherches évaluatives démontrant l'efficience de programmes (Palluy et coll., 2010), et ce, d'autant plus dans une perspective où, au Québec, une place plus formelle a récemment été accordée à l'éducation à la sexualité en milieu scolaire (MEES, 2018).

Les résultats obtenus par l'analyse des scores des enfants aux questionnaires d'évaluation du programme "On est encore des enfants! » (Duquet, 2017) sont concluants en ce qui concerne le développement des connaissances des contenus liés à la prévention de la sexualisation précoce. Cela pourrait être dû à divers critères de succès des évaluations de programmes identifiés par Smith, Schneider, Smith et Ananiadou (2004). Notamment, les programmes semblent plus concluants lorsqu'ils contiennent des directives claires destinées aux animateurs du programme - ce qui est tenté dans un effort que le programme soit clé en main pour les intervenants en charge de l'animer - ou lorsqu'ils sont destinés à des clientèles au primaire en comparaison avec ceux destinés à des clientèles plus âgées.

\section{Forces de l'étude}

L'une des forces de l'étude réside dans le choix d'analyses effectuées. En ce sens, les ANCOVAs à mesures répétées font en sorte qu'il est plus facile de déterminer si les résultats obtenus sont bel et bien attribuables aux effets sur les connaissances par rapport aux contenus du programme, en mesurant l'effet possible des variables de contrôle. Le choix d'un devis quasi expérimental est lui aussi l'un des éléments qui vérifie dans une meilleure portée que le programme soit bel et bien efficace, puisque l'augmentation des scores au post-test chez le groupe expérimental est davantage attribuable au fait d'avoir suivi les rencontres du programme, plutôt que d'avoir répondu au questionnaire à deux reprises (Bourassa et Rousseau, 2004; Donnelly et coll., 2016; Hébert et coll., 1999; Miller et coll., 2017; Verlaan et coll., 2005).

Nous concluons également que la production d'une recension des écrits au préalable de l'évaluation de programme, réalisée en fonction de la clientèle ciblée par ce dernier de même que par des recherches portant sur des programmes conçus dans le même domaine que celui sur lequel porte l'évaluation, semble une pratique gagnante pour guider les choix théoriques et méthodologiques subséquents. Par exemple, les mesures destinées aux enfants devraient davantage porter sur des items dichotomiques ou à 3 choix de réponses (Bourassa et Rousseau, 2004; Donnelly et coll., 2016; Hébert et coll., 1999; Verlaan et coll., 2005). Finalement, le Countenance Model (Stake, 1967), modèle d'évaluation de programme utilisé dans le cadre de cette étude, semble pertinent dans le contexte d'une évaluation de programme d'éducation à la sexualité au primaire, malgré qu'il ait à notre connaissance été utilisé dans le cadre de ce type d’évaluation pour la première fois. Rappelons que les autres études évaluatives réalisées dans des domaines similaires et utilisant ce modèle portaient sur des programmes d'éducation aux enfants et/ ou conduites en milieu scolaire (Chafel, 1981; Fatima et coll., 2016; Thanabalan et coll., 2015). 


\section{Limites de l'étude}

Labsence de théorie explicite à la base du programme est une limite importante de sa conception et, par conséquent, en restreint la portée de l'évaluation. Vu que des mesures de promotion de la santé efficaces s'appuient sur des programmes dont l'efficacité a été évaluée (Palluy, 2010), il appert de faciliter les démarches dévaluation en mentionnant les ancrages théoriques ayant guidé la conception des programmes. De plus, malgré que des intentions pédagogiques en lien avec chacune des thématiques soient incluses dans le programme, celles-ci ne mentionnent pas de façon claire les changements comportementaux attendus à la fin du programme. En ce sens, une évaluation complète du programme nécessiterait de préciser ces changements attendus chez la clientèle cible et de les mesurer dans le cadre d'une recherche évaluative. En effet, la théorie de changement d'un programme est une étape essentielle de toute évaluation des effets ou de l'impact d'un programme (UNFPA, 2015). D’ailleurs, plusieurs des évaluations de programmes destinés à des enfants dans le domaine de léducation à la sexualité ont adopté de telles mesures afin d'en évaluer l'efficacité (Miller et coll., 2017; Tremblay et Bégin, 2008; Verlaan et coll., 2005).

L'une des principales limites de l'étude est due à la méthode d'échantillonnage utilisée. Puisque létude n'est pas probabiliste, il se peut que les enfants ayant participé à l'étude ne présentent pas des caractéristiques semblables à l'ensemble de la population des enfants de 10 à 12 ans. Les effets du programme sur ces derniers peuvent donc être différents de ce qu'ils seraient chez d'autres participants. Cependant, le devis quasi expérimental avec mesures pré/post entre deux groupes de recherche ainsi que le respect des paramètres de l'analyse ont permis de pallier cette limite dans une certaine mesure en comparant les données recueillies auprès du groupe expérimental et celles recueillies auprès du groupe témoin.

En ce qui concerne les analyses effectuées, plusieurs limites méthodologiques se présentent. D’abord, les résultats des ANCOVAs à mesures répétées des $5^{\mathrm{e}}$ années démontrent une baisse de score au post-test pour les enfants du groupe témoin. Cela peut indiquer que l'instrument de mesure utilisé ne présente pas une bonne fidélité. Cependant, soulignons que la taille du groupe témoin de $5^{\mathrm{e}}$ année étant restreinte $(\mathrm{n}=11)$, il est probable que ce biais soit attribuable au petit nombre de participants.

Une dernière limite de létude concerne l'aspect selon lequel les effets du programme ont été évalués à court terme seulement. Le post-test étant mesuré immédiatement après la fin des rencontres et nayant pas été suivi d’une relance, il est primordial de considérer que cette méthodologie a favorisé les résultats. Ainsi, il est impossible de prévoir si les résultats se maintiendraient à moyen et à long termes. Dans cette mesure, il importe donc de ne pas tenir pour acquis que les résultats obtenus se maintiennent dans le temps. À ce sujet, d’autres programmes déducation à la sexualité destinés à des enfants du primaire ont utilisé des post-tests mesurés immédiatement après la fin du programme (Bourassa et Rousseau, 2004; Miller et coll., 2017; Tremblay et Bégin, 2008), mais malgré le fait que ce type de post-test soit pratique courante dans le domaine des évaluations de 
programmes en éducation à la sexualité, il est nécessaire d'exercer des évaluations régulières des programmes ainsi que des études pluriannuelles (UNFPA, 2015).

\section{Recherches futures et conclusion}

Dans le même sens que les limites exprimées à la présente recherche, il serait pertinent que les futures évaluations des effets de programmes d'éducation à la sexualité au primaire intègrent des variables comportementales à leurs mesures d'efficacité. Par exemple, Pacilli, Tomasetto et Cadinu (2016) ont établi que l'exposition à des images sexualisées affecte la mémoire de travail ainsi que le travail en mathématique d'enfants âgés de 8 à 10 ans. Dans le même ordre d'idées, lévaluation du "Relationship Building Intervention » (Miller et coll., 2017) a démontré que les enfants ayant suivi le programme, dont l'objectif est de promouvoir des relations positives entre pairs ainsi qu'une communauté inclusive au sein des groupes-classes scolaires, performent mieux sur le plan académique que les enfants du groupe contrôle. Ces deux études démontrent qu'il serait intéressant d'intégrer des mesures de la performance académique dans le cadre d'évaluations des effets de programmes d'éducation à la sexualité au primaire. Au Québec, l'éducation à la sexualité étant enseignée au travers de la planification de l'école et n'ayant par conséquent pas de matière ou de discipline lui étant consacrée (MEES, 2018b), de telles mesures renforcent les liens entre les programmes d'éducation à la sexualité et le Programme de formation de l'école québécoise, lequel permet la réalisation du projet éducatif des élèves québécois (MEES, 2019). De plus, les critères, les indicateurs ainsi que les méthodes de recherche élaborés dans le cadre des évaluations de programmes dans le domaine de léducation à la sexualité doivent davantage représenter des aspects positifs de la santé sexuelle, tels que des normes de genre positives ou des relations favorables à l'égalité des genres (UNFPA, 2015).

Par ailleurs, réaliser une recherche évaluative auprès d'un échantillon de plus grande taille permettrait une plus grande validité externe à la recherche. De plus, le maintien des connaissances acquises quant aux contenus du programme devrait être mesuré dans le cadre d'une relance, par exemple quelques mois après le programme. Finalement, mesurer l'acquisition de connaissances et de comportements attendus à l'égard du programme dans son entièreté serait plus que pertinent. Par exemple, dans le cas du programme « On est encore des enfants! », il serait intéressant de répliquer l'évaluation du programme en l'étudiant auprès d'une cohorte d'élèves de $5^{\mathrm{e}}$ année et de suivre cette dernière durant sa $6^{\mathrm{e}}$ année également. Cependant, toutes ces mesures permettant d'augmenter la rigueur méthodologique d'une étude évaluative demandent des efforts considérables que ne permettent pas toujours les contraintes données par un projet de recherche. Ultimement, la prise en compte du plus grand nombre de facteurs possibles dans l'évaluation des effets de programmes d'éducation à la sexualité permet d'émettre des recommandations visant à augmenter les formations aux enseignants ainsi que les budgets permettant de leur venir en aide dans l'implantation de l'éducation à la sexualité (UNFPA, 2015). Ces ressources sont d'autant plus pertinentes au sein 
de l'éducation à la sexualité au primaire, celle-ci étant à l'heure actuelle perçue comme étant moins bénéfique qu’au secondaire (MEES, 2018a).

\section{BIBLIOGRAPHIE}

Agence de la santé publique du Canada. (2008). Lignes directrices canadiennes pour l'éducation en matière de santé sexuelle. Consulté au https://www.canada.ca/fr/ sante-publique/services/rapports-publications/lignes-directrices-canadiennes-educationmatiere-sante-sexuelle/lignes-directrices-canadiennes-education-matiere-santesexuelle-4.html.

Bourassa, A. et Rousseau, J. (2004). Évaluation du programme de prévention des abus dans les classes de troisième année en Chaudière-Appalaches. Régie régionale de la santé et des services sociaux de Chaudière-Appalaches. Consulté au http://www.santecom. qc.ca/BibliothequeVirtuelle/Chaudiere-Appalaches/2895481628.pdf

Chafel, J. A. (1981). A preschool's experience with Stake's responsive evaluation model. Child Care Quarterly, 10(4), 307-317. https://doi.org/10.1007/bf01160686

Donnelly, J., Horn, R. R., Young, M. et Ivanescu, A. E. (2016). The effects of the Yes You Can! Curriculum on the sexual knowledge and intent of middle school students. Journal of School Health, 86(10), 759-765. https://doi.org/10.1111/josh.12429. Medline:27619767

Duquet, F. (2008a). Perceptions d'enfants du 3ième cycle primaire face à l'hypersexualisation et à la sexualisation précoce. Rapport préliminaire de recherche, Projet Outiller les jeunes face à l'hypersexualisation et Service aux collectivités de l'UQAM.

Duquet, F. (2008b). Perceptions des membres du personnel scolaire et du personnel de soutien face à l'hypersexualisation et la sexualisation précoce au primaire. Rapport de recherche préliminaire, Service aux collectivités de l'UQAM.

Duquet, F. (2017). On est encore des enfants! Programme de prévention de la sexualisation précoce pour des enfants de $3^{\text {ème }}$ cycle du primaire. Service des partenariats et du soutien à l'innovation, Université du Québec à Montréal. Consulté au https://hypersexualisation. uqam.ca/wp-content/uploads/sites/61/Programme-On-est-encore-des-enfants-2017. F.Duquet.compressé.pdf

Dussault, É. (2018). Évaluation du programme de prévention de la sexualisation précoce "On est encore des enfants! » destiné à des enfants âgés de 10 à 12 ans (Mémoire de maîtrise). Université du Québec à Montréal. Consulté au https://archipel.uqam. $\mathrm{ca} / 12361 /$

Fatima, G., Malik, M. et Abid, U. (2016). Early childhood special education program at the outcome phase: An evaluation from Stake's countenance model perspective. Bulletin of Education and Research, 38(2).

Fonds des nations unies pour la population (UNFPA). (2015). Évaluation des programmes d'éducation complète à la sexualité : une approche centrée sur les résultats en matière d'égalité des genres et d'autonomisation. Consulté au https://www.unfpa.org/sites/ default/files/pub-pdf/UNFPAEvaluation_FR.pdf

Haccoun, R. R. et McDuff, P. (2009). Attribution et causalité des effets. Dans V. Ridde et C. Dagenais (éds.), Approches et pratiques en évaluation de programme (pp. 109-124). Montréal: Presses de l'Université de Montréal. 
Hébert, M., Lavoie, F., Piché, C. et Poitras, M. (1999). Programme ESPACE : Évaluation des acquis des élèves. Rapport final de recherche présenté au Conseil québécois de la recherche sociale. Québec: Département d’orientation, d'administration et d'évaluation, Faculté des sciences de l'Éducation, Université Laval.

Mayne, J. (2015). Useful theory of change models. Canadian Journal of Program Evaluation, 30(2), 129-142. https://evaluationcanada.ca/system/files/cjpe-entries/ 30-2-119_0.pdf

Miller, C. F., Kochel, K. P., Wheeler, L. A., Updegraff, K. A., Fabes, R. A., Martin, C. L. et Hanish, L. D. (2017). The efficacy of a relationship building intervention in 5 th grade. Journal of School Psychology, 61, 75-88. https://doi.org/10.1016/j.jsp.2017.01.002. Medline:28259245

Ministère de l'Éducation et de l'Enseignement supérieur (MEES). (2017). Indices de défavorisation des écoles publiques, 2016-2017. Écoles primaires et secondaires. Consulté au http://www.education.gouv.qc.ca/fileadmin/site_web/documents/PSG/statistiques_ info_decisionnelle/Indices_PUBLICATION_20162017_final.pdf

Ministère de l'Éducation et de l'Enseignement supérieur (MEES). (2018a). Cahiers de formation et/ou présentations destinés aux enseignants pour le cours déducation à la sexualité. Consulté au http://www.education.gouv.qc.ca/fileadmin/site_web/documents/daai/17-249_Diffusion.pdf

Ministère de l'Éducation et de l'Enseignement supérieur (MEES). (2018b). Éducation à la sexualité. Consulté au http://www.education.gouv.qc.ca/enseignants/dossiers/ education-a-la-sexualite/

Ministère de l'Éducation et de l'Enseignement supérieur (MEES). (2019). Programme de formation de lécole québécoise. Consutlé au http://www.education.gouv.qc.ca/enseignants/ pfeq/

Organisation des Nations Unies pour l'éducation, la science et la culture (UNESCO). (2010). Principes directeurs internationaux sur l'éducation sexuelle : une approche factuelle à l'intention des établissements scolaires, des enseignants et des professionnels de l'éducation à la santé. Consulté au http://unesdoc.unesco.org/ images/0018/001832/183281f.pdf

Pacilli, M. G., Tomasetto, C. et Cadinu, M. (2016). Exposure to sexualized advertisements disrupts children's math performance by reducing working memory. Sex Roles, 74 (9-10), 389-398. https://doi.org/10.1007/s11199-016-0581-6

Palluy, J., Arcand, L., Choinière, C., Martin, C. et Roberge, M.-C. (2010). Réussite éducative, santé, bien-être : agir efficacement en contexte scolaire. Synthèse et recommandations. Québec: Institut national de santé publique du Québec. Consulté au https://www. inspq.qc.ca/pdf/publications/1065_ReussiteEducativeSanteBienEtre.pdf

Rossi, P. H., Lipsey, M. W. et Howard, E. F. (2004). Evaluation: A systematic approach (7th edition). Thousand Oaks, CA: Sage Publications.

Rush, E. et La Nauze, A. (2006). Letting children be children: Stopping the sexualisation of children in Australia. Consulté au http://www.tai.org.au/sites/default/files/DP93_8. pdf

Smith, J. D., Schneider, B. H., Smith, P. K. et Ananiadou, K. (2004). The effectiveness of whole-school antibullying programs: A synthesis of evaluation research. School Psychology Review, 33(4), 547-560. 
Stake, R. E. (1967). The countenance of educational evaluation. Teachers College Record, $68,523-540$.

Thanabalan, T. V., Siraj, S. et Alias, N. (2015). Evaluation of a digital story pedagogical module for the Indigenous learners using the stake countenance model. ProcediaSocial and Behavioral Sciences, 176(Suppl C), 907-914. https://doi.org/10.1016/j. sbspro.2015.01.557

Tremblay, C. et Bégin, H. (2008). Impact de l'intervention auprès d'enfants présentant des comportements sexuels problématiques. Revue québécoise de psychologie, 29(3), $15-26$.

Verlaan, P., Charbonneau, M.-N. et Turmel, F. (2005). Évaluation de l'implantation et des effets d'un programme de sensibilisation à l'agression indirecte auprès d'élèves du primaire. Nouveaux cahiers de la recherche en éducation, 8(2), 17-26. https://doi. org/10.7202/1017525ar

\section{PRÉSENTATION D'AUTEUR}

Éliane Dussault est étudiante au doctorat et chargée de cours au département de sexologie de l'Université du Québec à Montréal.

Francine Duquet est professeure au département de sexologie de l'Université du Québec à Montréal. 